Educar con tic: claves para pensar la ciudadanía digital y convivencia en la red. Páginas 53-64 en Revista de la Escuela de Ciencias de la EduCACIÓN, año 10, Número 9, enero a diciembre de 2014. ISSN 1851-6297. ISSN en LINEA 2362-3349.

\title{
EDUCAR CON TIC: CLAVES PARA PENSAR LA CIUDADANÍA DIGITAL Y CONVIVENCIA EN LA RED
}

\author{
Por Valentina Maltaneres \\ (Universidad Nacional de Rosario - Universidad Autónoma de Entre Ríos - \\ Universidad Tecnológica Nacional)", Argentina. \\ valentinamaltaneres@gmail.com
}

Recibido: 01/06/2014 Aceptado: 27/09/2014

\section{Resumen}

El trabajo analiza el oficio del educador a la luz de las problemáticas vinculadas a la inclusión de las nuevas tecnologías en la escuela secundaria. Relata una experiencia vivida por una docente participante del Programa de formación Laboratorio Pedagógico, del Ministerio de Educación de la Provincia de Santa Fe, cuyo material digital de su autoría fue dañado por un alumno/a de manera anónima. Luego de repasar conceptualizaciones sobre los Ilamados "nativos digitales" y del vínculo docente-alumno, avanza hacia el planteo de una nueva función del profesorado: educar para una cultura de la convivencia en las redes sociales.

\section{Palabras Clave}

TIC - Ciudadanía digital - Nativos digitales - Redes sociales - Convivencia.

\section{Abstract}

The paper analyzes the art of teaching in the light of the problems associated with the inclusion of new technologies in high school. It relates an experience of a teacher who participated in a training program "Pedagogical Laboratory", Ministry of Education of the Province of Santa Fe, and whose digital material created by herself, was damaged by a student anonymously. After reviewing conceptualizations of so-called

* Doctora en Psicología, docente e investigadora de la Facultad de Psicología de la UNR, de la UADER y UTN, en temáticas de educación, subjetividad y nuevas tecnologías. Coordina el programa de formación Tramas Digitales en la Dirección de Tecnología Educativa del Ministerio de Educación y anteriormente el Programa Laboratorio Pedagógico. 
Revista de la Escuela de Ciencias de la Educación, año 10, número 9, enero a diciembre de 2014. Páginas 53-64. ISSN 1851-6297. ISSN EN LINEA 2362-3349. EDUCAR CON TIC: CLAVES PARA PENSAR LA CIUDADANIA DIGITAL Y CONVIVENCIA en la red. Valentina Maltaneres.

"digital natives" and the teacher-student bond moves toward the proposition of a new role for teachers: educating for a culture of coexistence in social networks.

\section{Key Words}

New technologies of information and communication - Digital citizenship - Digital natives - Social networks - Coexistence.

Este escrito tiene la intención de plantear algunos interrogantes y líneas de reflexión en torno a la problemática de la inclusión de las nuevas tecnologías en la escuela secundaria, los vínculos que se establecen entre alumnos y docentes mediatizados por las mismas, los nuevos espacios de enseñanza y de aprendizaje que van más allá de las paredes de las escuelas y las características de los llamados "nativos digitales", que en definitiva implica hablar de nuevas matrices de constitución de la subjetividad en el siglo XXI.

Desde el año 2008 en la provincia de Santa Fe se han diseñado y llevado a cabo programas de formación docente para la integración de las TIC en las aulas de la Provincia de Santa Fe; se seleccionaron y formaron equipos de capacitadores en toda la provincia y se llevaron a cabo acciones de evaluación que posibilitaron conocer la complejidad de esta empresa, sus efectos en cuanto a logros y fracasos, sus alcances y limitaciones, su potencialidad y valor. La historia de la inclusión de las TIC en las escuelas secundarias de la provincia siguió un curso que inició con la llegada de equipamiento y el armado de los laboratorios de informática en las escuelas (Programas PROMSE; PROMEDU) hasta los actuales programas nacionales de entrega masiva de computadoras, bajo el modelo uno a uno (Plan IDE y Programa Conectar Igualdad) (1).

Lo que aconteció en este breve pero intenso lapso de seis años no sólo fue el aumento progresivo nacional y provincial de la inversión en equipamiento y conectividad. A nivel de la política educativa nacional se produjo un cambio paradigmático respecto a la forma de entender la enseñanza y el aprendizaje con TIC. De estar reducida en sus comienzos a unas pocas horas en la sala de computación (y por lo general a cargo del profesor de informática) pasó a tener una omnipresencia en todos los espacios escolares y en todas las materias. Vale decir, la inclusión de las nuevas tecnologías impactó directamente en la forma escolar tradicional. Toda la institución fue conmovida y las resistencias no faltaron, como tampoco los ejemplos de innovación y desarrollo profesional docente.

Gran parte de la tarea de formación docente consistió en construir sentido respecto a estas transformaciones que muchas veces fueron vividas como impuestas. Hubo que interrogar las prácticas pedagógicas, revisar el oficio de enseñar en el siglo XXI, volver la mirada sobre los alumnos y alumnas, e interpelar a la escuela en su conjunto. Y además... enseñar a los educadores 
Revista de la Escuela de Ciencias de la Educación, año 10, número 9, enero a diciembre de 2014. Páginas 53-64. ISSN 1851-6297. ISSN EN LINEA 2362-3349. EdUCAR CON TIC: CLAVES PARA PENSAR LA CIUDADANIA DIGITAL Y CONVIVENCIA en la Red. Valentina Maltaneres.

a hablar un nuevo lenguaje, el lenguaje digital, a manipular nuevos artefactos (netbooks, cañones, pizarras digitales) y a moverse en un entorno diferente, virtual, con una lógica hipertextual, en red, de no fácil acceso para ellos, los docentes, hijos de la cultura analógica, lineal, letrada.

Muchas fueron las experiencias acopiadas, y el conocimiento que aportaban progresivamente fue mejorando las estrategias y acciones en el territorio. La que aquí sigue es una pequeña muestra de lo que acontece en las aulas cuando se encuentran (¿o desencuentran?) educadores, jóvenes y nuevas tecnologías. Fue un suceso que sorprendió a los equipos del ministerio y dejó consternada a la docente involucrada. Y como suele suceder con este tipo de sucesos, resultan muy provechosos para el análisis y la reflexión, mucho más que las experiencias logradas.

La imagen que se muestra a continuación servirá como analizador de los problemas y desafíos que hoy implica educar con TIC.

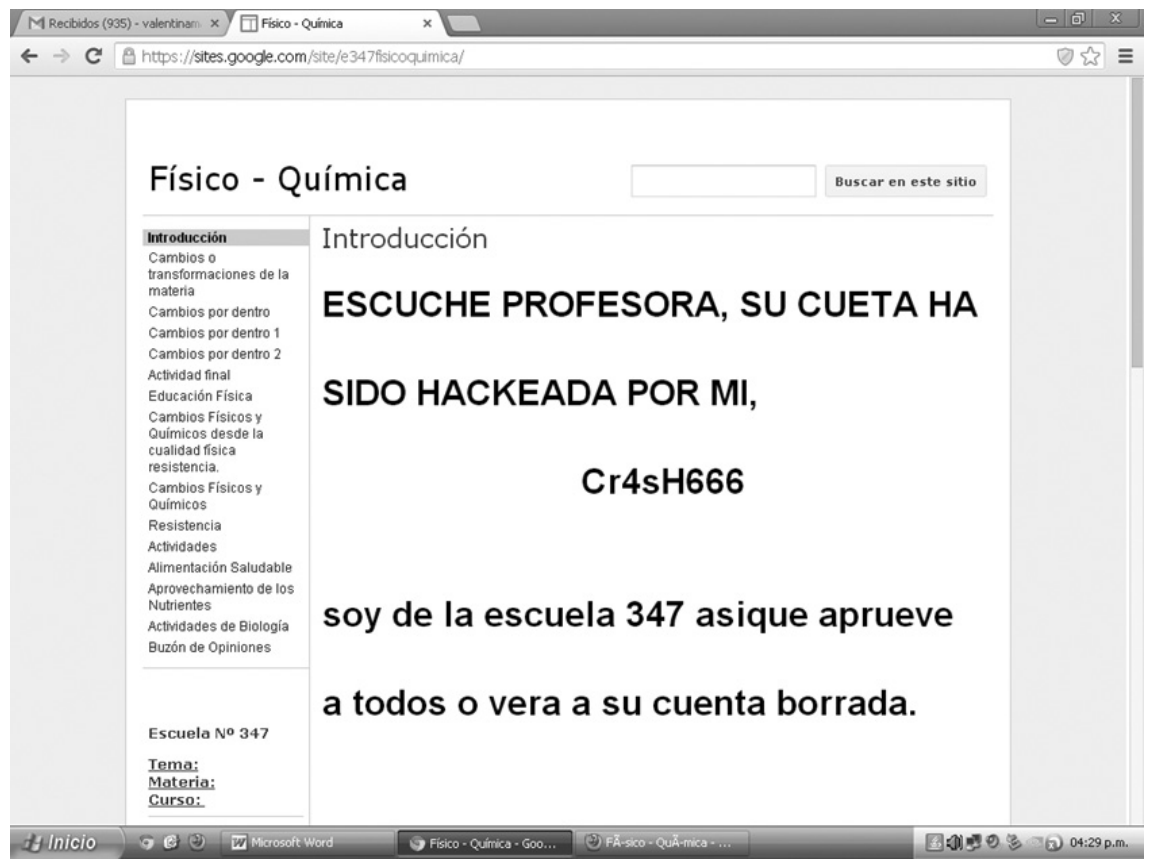

Figura 1: Imagen de la página de inicio del Site "Físico-Química"

Quizás muchos puedan identificar rápidamente de qué se trata. La imagen está sacada de una página de Internet (aun online) y pertenece a un sitio web (google site) creado por una docente que participó del programa de formación 
Revista de la Escuela de Ciencias de la Educación, año 10, número 9, enero a diciembre de 2014. Páginas 53-64. ISSN 1851-6297. ISSN EN LINEA 2362-3349. EDUCAR CON TIC: CLAVES PARA PENSAR LA CIUDADANIA DIGITAL Y CONVIVENCIA en la Red. Valentina Maltaneres.

Laboratorio Pedagógico. Resulta conveniente realizar unas breves referencias de este programa antes de comentar acerca de lo que aquí aconteció.

\section{El Programa Laboratorio Pedagógico}

Laboratorio Pedagógico (2) fue un dispositivo de formación y de producción implementado en Santa Fe, en el cual un grupo de docentes por escuela, acompañados por un referente TIC del Ministerio de Educación, crearon materiales virtuales de enseñanza durante un período de tres/cuatro meses, en forma intensiva, con la idea básica de renovar sus prácticas pedagógicas y generar oportunidades innovadoras de aprendizaje para los jóvenes de la escuela secundaria. Estos materiales creados fueron luego usados en las clases con los propios alumnos y pasaron a formar parte de un banco de recursos para uso de todos los docentes de la provincia. El proyecto se fundamentaba en la idea del docente productor, autor de sus propios materiales digitales de enseñanza y no mero usuario o consumidor de materiales creados por otros.

La creación de contenidos o materiales educativos digitales es un aspecto clave de la integración de TIC en el campo educativo. Una buena parte de la insatisfacción histórica de los docentes, y también de los estudiantes, para utilizarlos de manera habitual en sus prácticas se debió a la mala calidad de los materiales y recursos disponibles: siempre más referenciales que de otro tipo, la mayoría de las veces producidos en otros países y en otras lenguas, y, en general, poco flexibles para su utilización. Es decir, poco contextualizados a las necesidades concretas de un aula concreta. En general, sucedía como con los libros de texto que muchos profesores tienen que reajustar para sus intereses, debido a su propia naturaleza estática. Sin embargo, en el caso de los materiales educativos electrónicos este proceso de ajuste es mucho más complejo, o claramente imposible (Rodríguez Illera, Escofet y Azzato, 2005). De allí la importancia de generar espacios donde los docentes pudieran asumir un lugar de sujetos "prosumer", término acuñado por Alvin Toeffler (1980) y que designa esta posibilidad de convertirse en sujeto productor, autor, creador de bienes culturales. Como señala Cabero Almenara (2000), el profesor del siglo XXI "sin dudas" va a jugar un papel importante en el diseño de medios, materiales y recursos adaptados a las características de sus estudiantes, materiales que no sólo serán elaborados por él de forma independiente, sino en colaboración, tanto con el resto de compañeros involucrados en el proceso, como con otra serie de expertos. Desde esta perspectiva, el profesor deberá aprender a trabajar en equipo y en colaboración con otros profesionales.

Bajo estas ideas nació el proyecto como prueba piloto en el año 2009 y de allí en más se fue consolidando como una propuesta de gran impacto en las instituciones educativas. Prueba de ello son los cientos de materiales realizados de libre acceso (3); la cantidad de docentes formados que superó los 3000 educadores y la trascendencia a nivel nacional de la iniciativa. 
Revista de la Escuela de Ciencias de la Educación, año 10, número 9, enero a diciembre de 2014. Páginas 53-64. ISSN 1851-6297. ISSN EN LÍNEA 2362-3349. EDUCAR CON TIC: CLAVES PARA PENSAR LA CIUDADANIA DIGITAL Y CONVIVENCIA en la Red. Valentina Maltaneres.

En tanto capacitación en servicio, los docentes participantes contaron con licencia de horas para asistir a la formación y durante 12 encuentros revisaron la forma en que daban clases (y que entendían la propia disciplina), tomaron conciencia de las particularidades de sus alumno/as y aprendieron a usar las herramientas colaborativas gratuitas de la Web 2.0. Una de estas herramientas es google site, que permite diseñar sitios web en los que desplegar unidades didácticas. Estos sites integran texto, imagen, sonido, animaciones y transforman un contenido disciplinar lineal, estático y muchas veces poco atractivo para los jóvenes, en piezas multimediales, dinámicas, motivadoras, factibles de ser recorridas en diferentes sentidos y ritmos.

\section{Más allá de la enseñanza de la Físico-Química}

Dominar una herramienta de producción de contenidos (google site, exelearning o similares) requiere de muchas horas de dedicación y esfuerzo por parte de los docentes; acompañamiento cercano y altas dosis de tolerancia a la frustración y superación de temores. La imagen anterior muestra precisamente el trabajo en proceso de una profesora de Física y Química quien estaba construyendo un sitio sobre el tema "la materia y sus transformaciones".

Durante este proceso, un día, la profesora encontró que la página de inicio de su sitio en lugar de mostrar la introducción al tema, exhibía un mensaje escrito por un alumno/a: "ESCUCHE PROFESORA, SU CUETA HA SIDO HACKEADA POR MI, Cr4sH666 soy de la escuela 347 asique aprueve a todos o vera a su cuenta borrada".

El mensaje resultaba claro y contundente. Interpelaba a la docente en su lugar de saber y parecía reírse de su esfuerzo por dominar un código que le era extraño. Amenazaba con destruir por completo el trabajo de la profesora si ella no aprobaba a todos sus alumnos.

Quien profiere la amenaza desde el anonimato se autodesigna "Cr4sH666" y al mismo tiempo reconoce su filiación a la escuela 347. Podríamos preguntarnos ¿qué es lo que aquí irrumpe como problemática inesperada? ¿Se trata acaso de un problema viejo con nuevos ropajes?... De alguna forma el suceso invitaba a correr la mirada desde aquello que se enseña y la forma en que enseña (las prácticas de enseñanza, la didáctica, el currículo), hacia el vínculo docente-alumno; la autoridad pedagógica y la convivencia en el aula y en los nuevos entornos virtuales.

\section{De nativos digitales, bárbaros, hackers, adolescentes y algo más...}

En el año 2001 Marc Prensky escribía un artículo pionero llamado "Digital Natives, Digital Immigrants" (Nativos digitales, Inmigrantes digitales). Allí Prensky, refiriéndose a los debates sobre el decline de la educación en los Estados Unidos, llamaba la atención sobre un punto ignorado en aquel momento: el 
Revista de la Escuela de Ciencias de la Educación, año 10, número 9, enero a diciembre de 2014. Páginas 53-64. ISSN 1851-6297. ISSN EN LINEA 2362-3349. EDUCAR CON TIC: CLAVES PARA PENSAR LA CIUDADANIA DIGITAL Y CONVIVENCIA en la red. Valentina Maltaneres.

hecho que los estudiantes habían cambiado radicalmente y que no eran más aquellos para quienes el sistema educativo diseñó la enseñanza. Este cambio no era comparable a los cambios generacionales que siempre se suceden sino que se trataba de un gran salto o discontinuidad que ocurrió de la mano de la diseminación de la tecnología digital en las últimas décadas del Siglo XX. Los estudiantes a los que hacía mención Prensky representaban las primeras generaciones que crecieron con las nuevas tecnologías, que pasaron toda su vida rodeados de computadoras, videojuegos, reproductores de música digitales, cámaras de video, teléfonos celulares, y todos los demás juguetes y herramientas de la era digital.

Estos jóvenes habían pasado menos de 5.000 horas de su vida leyendo, pero más de 10.000 horas jugando videojuegos (sin mencionar las 20.000 horas viendo televisión). Los juegos de computadora, correo electrónico, Internet, teléfonos móviles y la mensajería instantánea eran parte integral de sus vidas. No era de extrañar que como resultado de este entorno se vieran transformados los procesos o patrones de pensamiento y sus estructuras. ¿Cómo designar a estos estudiantes? Prensky propone el término Digital Natives, sobre la idea que dichos estudiantes son "hablantes nativos" de la lengua digital, que es la lengua de las computadoras, los videojuegos e Internet. En contraposición a ellos estarían los adultos, Inmigrantes digitales, que si bien les puede atraer la tecnología, siempre conservaran el "acento" de su lengua de origen (predigital).

Su artículo es muy rico en señalar los problemas educativos que derivan del hecho que los docentes hablen una lengua predigital y deban enseñar a estudiantes que hablan una lengua nueva. Sus sugerencias apuntan a promover una verdadera transformación educativa tanto respecto a las metodologías como al contenido (aquello que hace a la transmisión del legado y al futuro), incluyendo con mayor énfasis el juego en las prácticas educativas.

Prensky acuñó un término que comenzó a expandirse y a ser utilizado en numerosas publicaciones. En nuestro contexto, Piscitelli (2009) retoma el concepto y habla de una nueva clase cognitiva, los "escribas del nuevo mundo", dedicando varias páginas a describir las características de estos jóvenes, sus consumos culturales o dieta cognitiva y la forma en que dichos consumos moldean la subjetividad. Más recientemente Balardini (2012) enlista una serie de atributos de esta "generación I" (4) tales como la visión positiva de la tecnología; la velocidad de procesamiento de información; los cambios en los parámetros de tiempo y espacio; la crisis y ruptura de linealidad (hipertexto); el procesamiento en paralelo (multitasking); el anonimato y la exploración de la identidad y la cibergrupalidad, entre otras. En otras palabras, como sintetiza Volnovich,

los "nativos digitales" aman la velocidad cuando de lidiar con la información se trata. Les encanta hacer varias cosas al mismo tiempo, casi todos ellos son multitasking y en muchos casos multimedia. Viven hiperconectados. Pueden oír la radio al tiempo que estudian en 
Revista de la Escuela de Ciencias de la Educación, año 10, número 9, enero a diciembre de 2014. Páginas 53-64. ISSN 1851-6297. ISSN EN LÍNEA 2362-3349. EDUCAR CON TIC: CLAVES PARA PENSAR LA CIUDADANIA DIGITAL Y CONVIVENCIA en la Red. Valentina Maltaneres.

un libro la lección de historia con la tele prendida, jugando a la play, hablando por el celular, chateando con medio mundo y comiendo pizza. Prefieren el universo gráfico al textual. Eligen el acceso aleatorio e hipertextual en lugar de la narrativa lineal. Funcionan mejor cuando operan en red, y lo que más aprecian es la gratificación constante y las recompensas permanentes que, por lo general, los incitan a desafíos de creciente complejidad. Pero, por sobre todo, prefieren jugar antes que estudiar. Su alimento verdadero son las golosinas digitales y no los alimentos convencionales. Pueden hackear la computadora más sofisticada por la noche y, por la mañana, reprobar el examen más sencillo de matemáticas (Volnovich, 2011, p. 2).

Otros términos aparecen cuando se trata de entender las mutaciones que dieron lugar a nuevas subjetividades: generación Einstein (Boschma y Groen, 2006), generación pulgar (Plant, 2008). Sin embargo, quizás otra denominación sea más útil para comprender el problema del sitio web hackeado por el alumno.

Alessandro Baricco nos propone la figura de "los bárbaros" (2006), una generación que vino a saquear el corazón de nuestra civilización y cuyo campamento, su capital, su palacio imperial es precisamente Google. Y ciertamente empezamos a entender no sólo lo que implica el saqueo, por ejemplo la indolencia y facilidad con que arrasan con la producción del docente, con su autoridad pedagógica, y los lugares del saber, sino también lo que implica arriesgarse a ingresar en el campamento de estos bárbaros. No por nada los educadores temen enfrentarse a sus alumnos/as usando tecnologías. Algunos ejemplos resultan muy claros al respecto. Se trata de testimonios de educadores que forman parte del material recabado en la investigación acreditada en UNR en el 2010, "La creación de contenidos educativos digitales en escuelas santafesinas. Resignificando el rol docente en la sociedad del conocimiento". Se analizaron narrativas escritas de los participantes antes y después de la experiencia de formación. Entre paréntesis están los seudónimos de los docentes:

Aprendimos, todo iba sobre ruedas...hasta que llegaron los nervios de la puesta en escena...y aquí aparecieron los fantasmas otra vez... Dar una clase para mí, después de 32 años de docencia no implica una situación estresante... ya no... Pero UNA CLASE NORMALLL... No con este bicho tecnológico que era capaz de tirar por la borda toda mi experiencia y seguridad... y claro, ahí desbarranqué... (Coqui).

Al comenzar la experiencia (capacitación) tenía dudas respecto a los resultados que podía lograr ya que no estaba acostumbrada a trabajar en la computadora preparando trabajos de este tipo e incluso sentía cierto temor de encontrarme en la sala de computación 
Revista de la Escuela de Ciencias de la Educación, año 10, número 9, enero a diciembre de 2014. Páginas 53-64. ISSN 1851-6297. ISSN EN LINEA 2362-3349. EDUCAR CON TIC: CLAVES PARA PENSAR LA CIUDADANIA DIGITAL Y CONVIVENCIA en la red. Valentina Maltaneres.

trabajando con los alumnos en ciertas situaciones que no supiera resolver...(Día).

Otro temor fue ir con mis alumnos a la sala de computación, pensaba que mi sitio no les iba a gustar o entenderlo. Pero la experiencia superó mis expectativas... (Tontin).

Admito que muchas veces sentí pánico y me paralicé en el temor y la ignorancia... Sin embargo la reacción de mis alumnos fue muy positiva... realmente me ayudaron (Juana III).

Posiblemente, la docente que nos ocupa, en tanto inmigrante digital, no tuvo en cuenta algunas cuestiones centrales: sus alumnos hablan la lengua digital y fácilmente aprenden a valerse de estrategias para superar barreras de seguridad, filtros y todo tipo de escollo informático que se interponga en la realización de sus propósitos, amparados en el anonimato que la red facilita. Es probable que este (o quizás esta) joven la haya simplemente "espiado" al momento de escribir la contraseña de su cuenta (lo que se conoce como hacking social) o quizás ella misma no cerró su sesión y dejó su máquina disponible. Esto sin olvidar que también puede contar con un programa muy simple de keylogger que le permite capturar todo lo que un usuario (en este caso su docente) ingresa a través del teclado y así obtener claves. De una u otra manera el problema en juego va más allá del uso pedagógico de las nuevas tecnologías: los jóvenes usan las TIC no sólo para surfear, para comunicarse, para jugar, para compartir toda clase de contenidos propios o ajenos. $Y$ hay algunos que incluso se valen de esas herramientas para interpelar a sus docentes, para poner en tensión las cuestiones del saber y del poder en el aula y la autoridad pedagógica, para ejercer violencia simbólica contra adultos y pares, para traspasar los límites de lo privado, para vulnerar derechos de otros.

Este aspecto tan sensible del oficio docente, la cuestión del vínculo con los alumnos y la legitimidad del lugar docente, según refiriera su capacitadora, no pudo ser trabajado por la profesora junto a su grupo. Sus acciones se orientaron a tratar de identificar a los responsables del hecho, sin suerte (trabajaban en la sala de computación donde incluso tenían acceso otros alumnos). El resultado final fue que la docente no intentó crear un nuevo sitio, hecho que habla del impacto que tuvo el evento en su subjetividad. Tampoco pudo sacar de Internet el sitio en construcción hackeado, ya que nunca pudo recuperar su contraseña, y por ende, el sitio permanece allí, online, invitando a la reflexión sobre qué implica hoy educar con TIC y en TIC.

Desde el equipo capacitador, no hubo instancias de profundización con la docente sobre lo acontecido. Y es que la formación focalizaba en el dominio y uso pedagógico de la herramienta. No contemplaba un más allá, es decir, avanzar sobre la cuestión de los procesos vinculares docente-alumno mediados por las nuevas tecnologías. Sin embargo, este simple evento no hizo más que 
Revista de la Escuela de Ciencias de la Educación, año 10, número 9, enero a diciembre de 2014. Páginas 53-64. ISSN 1851-6297. ISSN EN LÍNEA 2362-3349. EDUCAR CON TIC: CLAVES PARA PENSAR LA CIUDADANIA DIGITAL Y CONVIVENCIA en la Red. Valentina Maltaneres.

sacar a luz las condiciones de posibilidad para que puedan darse procesos de enseñanza y aprendizajes, en este caso de la física y de la química, en el aula.

\section{Educando para la ciudadanía digital}

Tim Berners-Lee y Robert Cailliau crearon la web alrededor de 1990. Durante las dos últimas décadas, Internet sufrió una evolución extraordinaria y asombrosa, apareciendo en 2004 el concepto de Web 2.0. Antes de la llegada de esta web, llamada Web colaborativa, se utilizaban páginas estáticas programadas en HTML (Hyper Text Markup Language) que no eran actualizadas frecuentemente. Además, la producción de contenidos estaba reservada a un grupo de expertos desarrolladores. La Web 2.0 vino a cambiar diametralmente las leyes de juego. Con ella vinieron las posibilidades de interacción y las redes sociales, y, lo más importante, la posibilidad de ser autor y productor de contenidos. Fueron los jóvenes quienes supieron apropiarse rápidamente de estas ventajas. Progresivamente Internet, comenzó a vislumbrarse como un espacio de ejercicio de los derechos, de convivencia y de participación. Desafortunadamente también mostró otra faceta: la de ser lugar de desconocimiento del otro, de violencia y discriminación. Esta doble naturaleza de las TIC ha sido suficientemente identificada y estudiada por numerosos autores (Espinar Ruiz y López Fernández, 2009; Burbules y Callister; 2006; Aviram, 2002).

Específicamente, surgieron interrogantes referidos a la cuestión de la convivencia en la red, las interacciones que se dan en la virtualidad y sus vínculos con el espacio real (la escuela, el hogar, los lugares de encuentro de los jóvenes). Nuevos términos aparecieron tales como ciberciudadanía; ciudadanía digital o ciudadanía comunicativa, los cuales ponen el énfasis en el manejo de algunas reglas escritas o normas sobre el comportamiento y el buen uso de las nuevas tecnologías y que otorgan una mayor importancia a la enseñanza para una ciudadanía responsable, que ayude a prevenir los riesgos que se pueden originar a partir del uso de las TIC, especialmente en los chicos y adolescentes (Galindo, 2008; Sánchez Carrero, 2009; Flores Fernández, 2009).

Czarny y Urbas (2007) describen cómo con el crecimiento progresivo de Internet y de sus posibilidades, se incrementó también la preocupación de parte de entidades vinculadas a la educación y a la preservación de los derechos de los niños y niñas por revisar y pensar en estrategias que llevaran a protegerlos y a educarlos para comprender las relaciones virtuales. En este marco se comenzó a hablar mundialmente de Safer Internet, o Internet segura. Este paradigma focalizaba en los peligros provenientes del "afuera" (delincuentes que usaban la web; pedófilos; redes de trata, etc.) y señalaba la necesidad de enseñar a los niños/as y adolescentes a cuidarse de tales peligros. Las autoras señalan las limitaciones de esta perspectiva y proponen migrar hacia el concepto de "uso responsable de las TIC", más apropiado por varios motivos. En principio, 
Revista de la Escuela de Ciencias de la Educación, año 10, número 9, enero a diciembre de 2014. Páginas 53-64. ISSN 1851-6297. ISSN EN LINEA 2362-3349. EDUCAR CON TIC: CLAVES PARA PENSAR LA CIUDADANIA DIGITAL Y CONVIVENCIA en la red. Valentina Maltaneres.

este concepto incluye el uso de otras tecnologías, no sólo Internet (celulares, cámaras digitales, etc.). Es decir, incluye aquellas tecnologías que hacen a la producción de audiovisuales, fotografías, que luego son socializadas a través de la Web.

A su vez incorpora la realidad de que la agresión no solo viene de "afuera", de extraños, sino que los mismos chicos/as y jóvenes, en muchas ocasiones, son los agresores. De allí que incluye fenómenos vinculados al anonimato, el maltrato como modalidad de interacción, el no respeto por la imagen del otro, a la intrusión en el ámbito de lo privado, entre otros. Esta perspectiva conduce a la pregunta acerca del lugar de los educadores y adultos responsables en la formación respecto de estos temas.

Finalmente, este enfoque incluye conceptos tales como ética, convivencia, privacidad, cuidado del cuerpo propio y del otro, respeto, para una interacción "cuidada" en los entornos virtuales, de la misma manera que se trabaja sobre los mismos ejes para favorecer la interacción "cara a cara". En síntesis, si acordamos que el aprendizaje de las pautas de ética y convivencia definen los vínculos entre las personas; estas pautas no deben ser diferentes para los vínculos que se establecen a través de lo virtual, sino que este "mundo" debe quedar dentro de las leyes sociales del respeto al otro, la tolerancia, el resguardo de la privacidad, la confidencialidad, etc. Como en la vida real, se trata de aprender progresivamente a entrar en relación con el otro, a colaborar, a reconocerlo como semejante pero también como un ser distinto. Y esto vale tanto si el otro es un par como si se trata del adulto docente.

Phillipe Meirieu (2006) introduce el concepto de aprendizaje de la alteridad, que resulta condición básica de la formación de ciudadanos para la vida democrática. Aprender a renunciar a ser el centro del mundo, metabolizar la violencia; ver al otro no como un peligro sino como alguien semejante a mí, pero distinto, son condiciones que requieren hoy ser analizadas e investigadas en las interacciones que ocurren mediatizadas por las nuevas tecnologías.

En este sentido y para el caso analizado, resulta necesario detener el tiempo de la clase de físico-química para indagar cómo los/las jóvenes entienden lo que allí aconteció en el espacio virtual. Vale decir, explorar la perspectiva de los sujetos en torno a esta cuestión, cómo perciben estos actos y el uso de los artefactos tecnológicos; qué significa para ellos lo público y lo privado en la red, cómo evalúan tales eventos; qué juicios morales realizan sobre los mismos (5). También es una oportunidad valiosa para que el docente se interrogue sobre el contenido del mensaje y el reclamo de que "todos sean aprobados"; sus estrategias de enseñanza y evaluación y sobre todo el vínculo que establece con sus alumnos/as.

La construcción de un site para la enseñanza podría haber sido en este caso la oportunidad para la construcción de una "cultura de la convivencia" en la escuela y en las redes sociales con este grupo de jóvenes estudiantes. No 
Revista de la Escuela de Ciencias de la Educación, año 10, número 9, enero a diciembre de 2014. Páginas 53-64. ISSN 1851-6297. ISSN EN LÍNEA 2362-3349. EDUCAR CON TIC: CLAVES PARA PENSAR LA CIUDADANIA DIGITAL Y CONVIVENCIA en la Red. Valentina Maltaneres.

pudo ser aprovechado como tal y quedó como una experiencia desafortunada en la historia de esta profesora. Sin embargo, el hecho no pasó desapercibido para la coordinación del programa. Todo lo contrario, señaló la necesidad de incorporar nuevos contenidos a la formación docente, en todos los niveles y modalidades del sistema educativo. En este sentido, el actual programa de formación ministerial denominado "Tramas Digitales" (6) contempla temáticas relativas al uso responsable de las nuevas tecnologías; el problema de la convivencia en las redes; la cuestión de los público y lo privado y el vínculo escuelafamilia. La formación para la ciudadanía digital empieza a estar en la agenda de los educadores santafesinos y enriquece el concepto de educación para la democracia. Más aún, avanzar en el fortalecimiento de la cultura democrática requiere investigar y promover la noción del "otro" como "semejante", lo cual implica el reconocimiento de las diferencias y, al mismo tiempo, la afirmación de su condición de igualdad de derechos. Sin el compromiso ético con el otro, no es posible la construcción del "nosotros" necesario para vivir en sociedad.

\section{Notas Bibliográficas}

(1) El Programa Conectar Igualdad nace con el decreto presidencial 459/10 y otorga una netbook a cada uno de los alumnos/as y docentes de educación secundaria, especial e institutos de formación docente, los capacita para el uso de dicha herramienta con el fin que se elaboren propuestas educativas que favorezcan la incorporación de las mismas en los procesos de enseñanza y aprendizaje. El programa es gestionado por un comité ejecutivo que incluye el ANSES; Jefatura de gabinete de ministros, Ministerio de Educación y Ministerio de Planificación federal.

(2) Resolución Ministerial $N^{\circ} 838 / 10$

(3) Pueden visualizarse materiales producidos en el programa en: https://sites.google.com/ site/santafelaboratoriopedagogico/sobre-el-proyecto. Actualmente el Ministerio trabaja en la configuración de un campus virtual donde se alojarán todas las producciones docentes.

(4) Generación I es otra de las denominaciones propuesta por Bill Gates donde "I" refiere a Internet, pero también a Información.

(5) He aquí un área de vacancia que dio lugar a un proyecto de investigación en curso sobre el tema, acreditado en la UNR: PSI 301 Significados de la violencia en la red entre estudiantes de escuelas santafesinas. Aportes al oficio de educar desde una ética del cuidado.

(6) Actualmente el Ministerio de Educación lleva adelante el Programa de Formación Tramas Digitales destinado a docentes de todos los niveles y modalidades del sistema educativo. Resolución Ministerial № 1696/13.

\section{Referencias bibliográficas}

- Aviram, R. (2002). ¿Conseguirá la educación domesticar a las TIC? II Congreso europeo de TIC en la educación y la ciudadanía. Barcelona, 26,27 y 28 de junio. Documento recuperado de http://tecnologiaedu.us.es/nweb/htm/pdf/pon1.pdf 
Revista de la Escuela de Ciencias de la Edducación, año 10, número 9, enero a diciembre de 2014. PÁginas 53-64. ISSN 1851-6297. ISSN EN LINEA 2362-3349. EdUCAR CON TIC: CLAVES PARA PENSAR LA CIUDADANIA DIGITAL Y CONVIVENCIA en la Red. Valentina Maltaneres.

- $\quad$ Balardini, S. (2006, Julio). Subjetividades Juveniles y Tecnocultura. En Panel: Tecnología y subjetividad juveniles. Impacto y transformaciones de la cultura escolar ante la inclusión de las tecnologías de la información y la comunicación. Centro Cultural del Teatro Municipal General San Martín.

- $\quad$ Baricco, A. (2008). Los Bárbaros, ensayo sobre la mutación. Barcelona: Anagrama 2008. Colección Argumentos.

- Boschma, J \& Groen, I (2006). Generation Einstein: smart, social and superfast. Communicating with Young People in the 21st Century, Pearson Education Benelux, Schiedam.

- $\quad$ Burbules, N. y Callister, T (2001). Educación: Riesgos y Promesas de las Nuevas Tecnologías de la Información. Buenos Aires: Ediciones Granica.

- Cabero Almenara, J (2000). El rol del profesor en los nuevos entornos de comunicación. Agenda Académica, 7(1), 41-57.

- Czarny, M y Urbas, A. (2007). De qué hablamos cuando decimos "Uso responsable de las TIC por parte de niños, niñas y adolescentes". Colección EducAr CD 27. Ministerio de Educación de la Nación.

- $\quad$ Espinar Ruiz, E; López Fernández, C (2009). Jóvenes y adolescentes ante las nuevas tecnologías: percepción de riesgos. Athenea Digital. Revista de Pensamiento e Investigación Social. 1(20).

- Flores Fernández, J. (2009). Uso seguro de Internet y ciudadanía digital responsable. Recuperado de http://www.pantallasamigas.net/proteccion-infancia-consejos-articulos/ uso-seguro-de-internet-y-ciudadania-digital-responsable.shtm.

- Galindo J.A (2009). Ciudadanía digital. Revista Signo y Pensamiento, volumen XXVIII (54), 164-173.

- Meirieu, P. (2006). El significado de educar en un mundo sin referencias. Conferencia para los Institutos de Formación Docente. Ministerio de Educación de la Nación. Recuperado de http://www.me.gov.ar/curriform/publica/meirieu_final.pdf.

- Piscitelli, A. (2009). Nativos Digitales: Dieta cognitiva, inteligencia colectiva y arquitecturas de participación. Buenos Aires: Editorial Santillana. Colección Aula XXI.

- $\quad$ Plant, S (2008). On the mobile: the effects of mobile telephones on social and individual life. At the Wayback Machine (archived 25 June 2008).

- Prensky, M (2001). Digital Natives, Digital Immigrants On the Horizon MCB University Press, Vol. 9 No. 5.

- $\quad$ Rodríguez Illera, J; Escofet, A; Azzato, M (2005). Un sistema abierto para la creación de contenidos educativos digitales. RED: Revista de Educación a Distancia, №. Extra 4, 2005 Guadalajara, Mexico.

- Volnovich, J. C. Los jóvenes y sus "golosinas digitales". Diario Página 12. 14 de julio de 2011. Recuperado de http://www.pagina12.com.ar/diario/psicologia/9-172181-2011-07-14. html. 